ESAIM: PROCEEDINGS AND SURVEYS, September 2014, Vol. 45, p. 168-177

J.-S. Dhersin, Editor

\title{
UNIFORM ASSYMPTOTICS IN THE AVERAGE CONTINUOUS CONTROL OF PIECEWISE DETERMINISTIC MARKOV PROCESSES : VANISHING APPROACH $*, * *$
}

\author{
Dan Goreac ${ }^{1}$ And OAna-Silvia Serea ${ }^{2}$
}

\begin{abstract}
We prove a uniform Abelian result for controlled systems with piecewise deterministic Markov dynamics : the existence of a uniform limit for value functions with discounted costs as the discount factor decreases to zero implies the existence of a (uniform) value function with long time average cost. The result is independent of dissipativity properties of the control system.
\end{abstract}

AMS Classification: 49L25, 60J25, 93E20

Keywords: piecewise deterministic Markov processes, long run average optimal control

\section{INTRODUCTION}

For sequences of bounded real numbers $\left(x_{n}\right)_{n \geq 1}$, Hardy and Littlewood (cf. [17]) have proved that the convergence of the Cesàro means $\left(\frac{1}{n} \sum_{i=1}^{n} x_{i}\right)_{n \geq 1}$ is equivalent to the convergence of their Abel means $\left(\delta \sum_{i=1}^{\infty}(1-\delta)^{i} x_{i}\right)$. This result has been generalized by Feller (cf. [12], XIII.5) to the case of uncontrolled deterministic dynamics in continuous time, [2] to deterministic controlled dynamics, etc. A further generalization (cf. [19]) allows the limit value function with respect to a system governed by controlled deterministic dynamics to depend on the initial data. In the Brownian diffusion setting, similar results have been obtained in [4].

Piecewise deterministic Markov processes (PDMP) have been introduced by Davis [8], [10]. The literature on optimal control topics in connection to these processes is extremely wide ( [9], [20], [1], [11], [13], etc.). However, the cited papers deal mainly with infinite-horizon, discounted costs. The literature on control problems with long time average cost is less rich. To the best of our knowledge, the first papers to deal with average costs for impulsive control problems were [5] and [14]. In the framework of continuous control, the first papers on the long time average cost are [7] and [6].

Controlled piecewise deterministic Markov processes are given by their local characteristics: a vector field $f: \mathbb{R}^{N} \times U \rightarrow \mathbb{R}^{N}$ that determines the motion between two consecutive jumps, a jump rate $\lambda: \mathbb{R}^{N} \times U \rightarrow \mathbb{R}_{+}$ and a transition measure $Q: \mathbb{R}^{N} \times U \rightarrow \mathcal{P}\left(\mathbb{R}^{N}\right)$. The set $U$ is a compact metric space (the control space) and $\mathbb{R}^{N}$ is the state space, for some $N \geq 1$. We denote by $X^{x, u}$ the trajectories associated to local characteristics

* The work of the first author has been partially supported by the French National Research Agency ANR PIECE.

** The work of the second author has been partially supported by the European project SADCO - Sensitivity Analysis for Deterministic Controller Design, (Marie Curie ITN-264735) and by the ANR Jeudy: ANR-10-BLAN 0112.

${ }^{1}$ Université Paris-Est, LAMA, UMR8050, 5, boulevard Descartes, Cité Descartes, Champs-sur-Marne, 77454 Marne-la-Vallée, France.

${ }^{2}$ Univ. Perpignan Via Domitia, LAboratoire de Mathématiques et PhySique, EA 4217, F-66860 Perpignan, France

(C) EDP Sciences, SMAI 2014 
$(f, \lambda, Q)$ issued from $x$ and controlled by $u$. The construction of controlled PDMPs and basic assumptions are recalled in section 2 .

Whenever $\delta, t>0$, the $\delta$-discounted value function is given by

$$
v^{\delta}(x)=\inf _{u} \delta \mathbb{E}\left[\int_{0}^{\infty} e^{-\delta r} g\left(X_{r}^{x, u}\right) d r\right]
$$

and the time averaged value function up to $t$ by

$$
V_{t}(x)=\inf _{u} \frac{1}{t} \mathbb{E}\left[\int_{0}^{t} g\left(X_{r}^{x, u}\right) d r\right],
$$

for all $x \in \mathbb{R}^{N}$. Following the idea of [19], we propose a sufficient criterion for the existence of a (uniform) limit value function for continuous control problems with long run average costs using a uniform vanishing technique. The vanishing technique has also been employed by [7]. However, the formulation of the long time average control problem is slightly different in our case. The cost functional in [7] is given by a lim sup formulation (thus giving an inf/sup value function) :

$$
\inf _{u} \limsup _{t \rightarrow \infty} \frac{1}{t} \mathbb{E}\left[\int_{0}^{t} g\left(X_{r}^{x, u}\right) d r\right]=\inf _{T \rightarrow \infty} \inf _{u} \sup _{t \geq T} \frac{1}{t} \mathbb{E}\left[\int_{0}^{t} g\left(X_{r}^{x, u}\right) d r\right] .
$$

We partially extend the results of [19] to continuous control of piecewise deterministic Markov process. In our main result (Theorem 4.1), we prove that, whenever $\lim _{\delta \rightarrow 0} v^{\delta}$ exists uniformly on the state space, the limit value $\lim _{t \rightarrow \infty} V_{t}$ also exists (which gives a sup/inf long time average value function). Moreover, this limit is uniform in space and the limit value functions coincide. This result can be seen of a counterpart of [7]. Our approach (implicitely) relies on the theory of viscosity solutions for Hamilton-Jacobi integro-differential systems.

In the first section (section 2), we recall the standard assumptions and the construction of PDMP. Using the so-called "shaking of coefficients" method for PDMPs (see [18], [16]), we give some technical tools in section 3. The main result is stated and proven in section 4.

\section{Controlled PDMPS}

We consider $U$ (the control space) to be a compact subspace of a metric space $\mathbb{R}^{d}$ and $\mathbb{R}^{N}$ be the state space, for some $N, d \geq 1$.

Piecewise deterministic control processes have been introduced by Davis [10]. Such processes are given by their local characteristics: a vector field $f: \mathbb{R}^{N} \times U \rightarrow \mathbb{R}^{N}$ that determines the motion between two consecutive jumps, a jump rate $\lambda: \mathbb{R}^{N} \times U \rightarrow \mathbb{R}_{+}$and a transition measure $Q: \mathbb{R}^{N} \times U \rightarrow \mathcal{P}\left(\mathbb{R}^{N}\right)$. We denote by $\mathcal{B}\left(\mathbb{R}^{N}\right)$ the Borel $\sigma$-field on $\mathbb{R}^{N}$ and $\mathcal{P}\left(\mathbb{R}^{N}\right)$ the family of probability measures on $\mathbb{R}^{N}$. For every $A \in \mathcal{B}\left(\mathbb{R}^{N}\right)$, the function $(x, u) \mapsto Q(x, u, A)$ is assumed to be measurable and, for every $(x, u) \in \mathbb{R}^{N} \times U, Q(x, u,\{x\})=0$.

We summarize the construction of controlled piecewise deterministic Markov processes (PDMP). We let $\mathbb{L}^{0}\left(\mathbb{R}^{N} \times \mathbb{R}_{+} ; U\right)$ denote the space of $U$-valued Borel measurable functions defined on $\mathbb{R}^{N} \times \mathbb{R}_{+}$. Whenever $u \in \mathbb{L}^{0}\left(\mathbb{R}^{N} \times \mathbb{R}_{+} ; U\right)$ and $\left(t_{0}, x_{0}\right) \in \mathbb{R}_{+} \times \mathbb{R}^{N}$, we consider the ordinary differential equation

$$
\left\{\begin{array}{l}
d \Phi_{t}^{t_{0}, x_{0}, u}=f\left(\Phi_{t}^{t_{0}, x_{0}, u}, u\left(x_{0}, t-t_{0}\right)\right) d t, t \geq t_{0} \\
\Phi_{t_{0}}^{t_{0}, x_{0}, u}=x_{0}
\end{array}\right.
$$

We choose the first jump time $T_{1}$ such that the jump rate $\lambda\left(\Phi_{t}^{0, x_{0}, u}, u\left(x_{0}, t\right)\right)$ satisfies

$$
\mathbb{P}\left(T_{1} \geq t\right)=\exp \left(-\int_{0}^{t} \lambda\left(\Phi_{s}^{0, x_{0}, u}, u\left(x_{0}, s\right)\right) d s\right) .
$$


The controlled piecewise deterministic Markov processes (PDMP) is defined by

$$
X_{t}^{x_{0}, u}=\Phi_{t}^{0, x_{0}, u}, \text { if } t \in\left[0, \tau_{1}\right) .
$$

The post-jump location $Y_{1}$ has $Q\left(\Phi_{\tau}^{0, x_{0}, u}, u\left(x_{0}, \tau\right), \cdot\right)$ as conditional distribution given $\tau_{1}=\tau$. Starting from $Y_{1}$ at time $\tau_{1}$, we select the inter-jump time $\tau_{2}-\tau_{1}$ such that

$$
\mathbb{P}\left(\tau_{2}-\tau_{1} \geq t / \tau_{1}, Y_{1}\right)=\exp \left(-\int_{\tau_{1}}^{\tau_{1}+t} \lambda\left(\Phi_{s}^{\tau_{1}, Y_{1}, u}, u\left(Y_{1}, s-\tau_{1}\right)\right) d s\right) .
$$

We set

The post-jump location $Y_{2}$ satisfies

$$
X_{t}^{x_{0}, u}=\Phi_{t}^{\tau_{1}, Y_{1}, u}, \text { if } t \in\left[\tau_{1}, \tau_{2}\right) .
$$

$$
\mathbb{P}\left(Y_{2} \in A / \tau_{2}, \tau_{1}, Y_{1}\right)=Q\left(\Phi_{\tau_{2}}^{\tau_{1}, Y_{1}, u}, u\left(Y_{1}, \tau_{2}-\tau_{1}\right), A\right),
$$

for all Borel set $A \subset \mathbb{R}^{N}$. And so on.

Throughout the paper, unless stated otherwise, we assume the following:

(A1) The function $f: \mathbb{R}^{N} \times U \longrightarrow \mathbb{R}^{N}$ is uniformly continuous on $\mathbb{R}^{N} \times U$ and there exists a positive real constant $C>0$ such that

$$
|f(x, u)-f(y, u)| \leq C|x-y|, \text { and }|f(x, u)| \leq C,
$$

for all $x, y \in \mathbb{R}^{N}$ and all $u \in U$.

(A2) The function $\lambda: \mathbb{R}^{N} \times U \longrightarrow \mathbb{R}_{+}$is uniformly continuous on $\mathbb{R}^{N} \times U$ and there exists a positive real constant $C>0$ such that

for all $x, y \in \mathbb{R}^{N}$ and all $u \in U$.

$$
|\lambda(x, u)-\lambda(y, u)| \leq C|x-y|, \text { and } \lambda(x, u) \leq C,
$$

(A3) The function $Q: \mathbb{R}^{N} \times U \longrightarrow \mathcal{P}\left(\mathbb{R}^{N}\right)$ is continuous on $\mathbb{R}^{N} \times U$ and for each bounded uniformly continuous function $h \in B U C\left(\mathbb{R}^{N}\right)$, there exists a continuous function $\eta_{h}: \mathbb{R} \longrightarrow \mathbb{R}$ such that $\eta_{h}(0)=0$ and

$$
\sup _{u \in U}\left|\int_{\mathbb{R}^{N}} h(z) Q(x, u, d z)-\int_{\mathbb{R}^{N}} h(z) Q(y, u, d z)\right| \leq \eta_{h}(|x-y|) .
$$

(A4) For every $x \in \mathbb{R}^{N}$ and every decreasing sequence $\left(\Gamma_{n}\right)_{n \geq 0}$ of subsets of $\mathbb{R}^{N}$,

$$
\inf _{n \geq 0} \sup _{u \in U} Q\left(x, u, \Gamma_{n}\right)=\sup _{u \in U} Q\left(x, u, \cap_{n} \Gamma_{n}\right)
$$

and

$$
\inf _{n \geq 1} \sup _{x \in \mathbb{R}^{N}, u \in U} Q\left(x, u, \mathbb{R}^{N} \backslash \bar{B}(x, n)\right)=0 .
$$

Remark 2.1. 1. Assumption (A3) can be somewhat weakened by imposing

(A3') For each bounded uniformly continuous function $h \in B U C\left(\mathbb{R}^{N}\right)$, there exists a continuous function $\eta_{h}: \mathbb{R} \longrightarrow \mathbb{R}$ such that $\eta_{h}(0)=0$ and

$$
\sup _{u \in U}\left|\lambda(x, u) \int_{\mathbb{R}^{N}} h(z) Q(x, u, d z)-\lambda(y, u) \int_{\mathbb{R}^{N}} h(z) Q(y, u, d z)\right| \leq \eta_{h}(|x-y|) .
$$

It is obvious that whenever one assumes (A3) and $\lambda(\cdot)$ is bounded, the assumption A3' holds true. Moreover, all the proofs in this paper can be obtained (with minor changes) when A3' replaces A3. 
Similarly, one can replace in (A4) $Q$ by $\lambda Q$.

2. The assumptions (A1-A3) are quite standard when dealing with viscosity theory in PDMP. They appear under this form in [20] and are needed to infer the uniform continuity of the value function. The assumption (A4) is needed in the Appendix of [16] to provide stability properties of viscosity solutions. Roughly speaking, (A4b) states that the probability of exiting the ball centered at the initial point is zero as the radius increases to $\infty$. The main linearization result is independent of (A4) as soon as stability for the associated system is provided.

3. To apply the "shaking of coefficients" method of [18] (see also [3]), we need to strengthen (A3) (or (A3')) and assume

(B) For each bounded uniformly continuous function $h \in B U C\left(\mathbb{R}^{N}\right)$, there exists a continuous function $\eta_{h}: \mathbb{R} \longrightarrow \mathbb{R}$ such that $\eta_{h}(0)=0$ and

$$
\sup _{u^{1} \in U, u^{2} \in \bar{B}(0,1)}\left|\int_{\mathbb{R}^{N}} h\left(z-u^{2}\right) Q\left(x+u^{2}, u^{1}, d z\right)-\int_{\mathbb{R}^{N}} h\left(z-u^{2}\right) Q\left(y+u^{2}, u, d z\right)\right| \leq \eta_{h}(|x-y|) .
$$

For further details on these assumptions as well as for connections with stochastic gene networks, the reader is referred to [15] and [16].

\section{Some teChnicAl IngREDiEnts}

Unless stated otherwise, the cost function $g: \mathbb{R}^{N} \longrightarrow \mathbb{R}$ is assumed to be bounded and Lipschitz-continuous. Moreover, we may assume that $0 \leq g(x) \leq 1$, for all $x \in \mathbb{R}^{N}$.

For every finite time horizon $t>0$, let us introduce the average value function by setting

$$
V_{t}(x)=\inf _{u \in \mathbb{L}^{0}\left(\mathbb{R}^{N} \times \mathbb{R}_{+} ; U\right)} \frac{1}{t} \mathbb{E}\left[\int_{0}^{t} g\left(X_{s}^{x, u}\right) d s\right],
$$

for all $x \in \mathbb{R}^{N}$.

For every $\delta>0$, the $\delta$-discounted value function is given by

$$
v^{\delta}(x)=\inf _{u \in \mathbb{L}^{0}\left(\mathbb{R}^{N} \times \mathbb{R}_{+} ; U\right)} \delta \mathbb{E}\left[\int_{0}^{\infty} e^{-\delta t} g\left(X_{t}^{x, u}\right) d t\right]
$$

for all $x \in \mathbb{R}^{N}$. It is known (cf. [20]) that $v^{\delta}$ is the unique bounded uniformly continuous viscosity solution of

$$
\delta v^{\delta}(x)-\delta g(x)+H\left(x, \nabla v^{\delta}(x), v^{\delta}\right)=0,
$$

for all $x \in \mathbb{R}^{N}$, where the Hamiltonian $H$ is given by

$$
H(x, p, \psi)=\sup _{u \in U}\left\{-\langle f(x, u), p\rangle-\lambda(x, u) \int_{\mathbb{R}^{N}}(\psi(z)-\psi(x)) Q(x, u, d z)\right\} .
$$

Under the assumptions (A1-4) and (B), using the so-called "shaking of coefficients" method (introduced in [18] for Brownian diffusions), there exists a family of regular subsolutions of (1) denoted $\left(v_{\varepsilon}^{\delta}\right)_{\varepsilon>0}$ such that

$$
\lim _{\varepsilon \rightarrow 0} \sup _{x \in \mathbb{R}^{N}}\left|v_{\varepsilon}^{\delta}(x)-v^{\delta}(x)\right|=0 .
$$

For further details, the reader is referred to [16], eq. (11) and (15).

In particular, this allows one to obtain monotonicity results for the discounted value functions. 
Proposition 3.1. 1. For every $T_{0}>0$, every initial data $x \in \mathbb{R}^{N}$ and every admissible control $u \in \mathbb{L}^{0}\left(\mathbb{R}^{N} \times \mathbb{R}_{+} ; U\right)$, one has

$$
\liminf _{\delta \rightarrow 0} v^{\delta}(x) \leq \liminf _{\delta \rightarrow 0} \mathbb{E}\left[v^{\delta}\left(X_{T_{0}}^{x, u}\right)\right] .
$$

2. For every $x \in \mathbb{R}^{N}, T_{0}>0$, every $\delta>0$ and every admissible control $u \in \mathbb{L}^{0}\left(\mathbb{R}^{N} \times \mathbb{R}_{+} ; U\right)$,

$$
\mathbb{E}\left[v^{\delta}\left(X_{T_{0}}^{x, u}\right)\right] \leq \mathbb{E}\left[\delta \int_{0}^{\infty} e^{-\delta t} g\left(X_{T_{0}+t}^{x, u}\right) d t\right] .
$$

Proof. For the first assertion, we begin by fixing $\delta>0$. For $\varepsilon>0$, we apply Itô's formula (cf. Theorem 31.3 in [10]) to $e^{-\delta \cdot} v_{\varepsilon}^{\delta}\left(X^{x, u}\right)$ on $\left[0, T_{0}\right]$ (where $v_{\varepsilon}^{\delta}$ satisfy 3 ) to get

$$
\mathbb{E}\left[e^{-\delta T_{0}} v_{\varepsilon}^{\delta}\left(X_{T_{0}}^{x, u}\right)\right]=v_{\varepsilon}^{\delta}(x)+\mathbb{E}\left[\int_{0}^{T_{0}} e^{-\delta t}\left(\mathcal{U}^{u_{t}} v_{\varepsilon}^{\delta}\left(X_{t}^{x, u}\right)-\delta v_{\varepsilon}^{\delta}\left(X_{t}^{x, u}\right)\right) d t\right] .
$$

By abuse of notation, we let

$$
\mathcal{U}^{u_{t}} \phi\left(X_{t}^{x, u}\right)=\mathcal{U}^{u\left(X_{\tau_{i}}^{x, u}, t-\tau_{i}\right)} \phi\left(X_{t}^{x, u}\right), \text { whenever } \tau_{i} \leq t<\tau_{i+1},
$$

where $\tau_{i}$ are the jump times appearing in section 2. Since the functions $v_{\varepsilon}^{\delta}$ are (regular) subsolutions of (1), one gets

$$
e^{-\delta T_{0}} \mathbb{E}\left[v_{\varepsilon}^{\delta}\left(X_{T_{0}}^{x, u}\right)\right] \geq v_{\varepsilon}^{\delta}(x)-\delta \mathbb{E}\left[\int_{0}^{T_{0}} e^{-\delta t} g\left(X_{t}^{x, u}\right) d t\right] .
$$

Taking the limit as $\varepsilon \rightarrow 0$, the equality (3) yields

$$
e^{-\delta T_{0}} \mathbb{E}\left[v^{\delta}\left(X_{T_{0}}^{x, u}\right)\right] \geq v^{\delta}(x)-\delta \mathbb{E}\left[\int_{0}^{T_{0}} e^{-\delta t} g\left(X_{t}^{x, u}\right) d t\right] .
$$

The conclusion follows by taking liminf as $\delta \rightarrow 0$ and recalling that $0 \leq g \leq 1$.

The proof of the second assertion is quite similar. For $S>T_{0}$, one applies Itô's formula to $e^{-\delta \cdot} v_{\varepsilon}^{\delta}\left(X^{x, u}\right)$ on $\left[T_{0}, S\right]$, then lets $S \rightarrow \infty$ and $\varepsilon \rightarrow 0$. Our proposition is now complete.

The second ingredient is the following.

Proposition 3.2. If $1>\varepsilon>0$, then, for all initial condition $x \in \mathbb{R}^{N}$, all $t>0$ and all $u \in \mathbb{L}^{0}\left(\mathbb{R}^{N} \times \mathbb{R}_{+} ; U\right)$ for which

$$
\frac{1}{t} \mathbb{E}\left[\int_{0}^{t} g\left(X_{r}^{x, u}\right) d r\right] \leq V_{t}(x)+\frac{\varepsilon}{3},
$$

one is able to find some $0 \leq T \leq t\left(1-\frac{\varepsilon}{3}\right)$ such that

$$
\frac{1}{s} \mathbb{E}\left[\int_{T}^{T+s} g\left(X_{r}^{x, u}\right) d r\right] \leq V_{t}(x)+\varepsilon,
$$

for every $0<s \leq t-T$.

Proof. One introduces the set

$$
A:=\left\{s \in(0, t]: \frac{1}{s} \mathbb{E}\left[\int_{0}^{s} g\left(X_{r}^{x, u}\right) d r\right]>V_{t}(x)+\varepsilon\right\} .
$$


If the set is empty, $T=0$ satisfies the conditions. Otherwise, one introduces $T:=\sup \{s: s \in A\}$. It is clear that $T \leq t\left(1-\frac{\varepsilon}{3}\right)$. Indeed, whenever $s \in\left[t\left(1-\frac{\varepsilon}{3}\right), t\right]$,

$$
\frac{1}{s} \mathbb{E}\left[\int_{0}^{s} g\left(X_{r}^{x, u}\right) d r\right] \leq \frac{1}{1-\frac{\varepsilon}{3}}\left(V_{t}(x)+\frac{\varepsilon}{3}\right) \leq V_{t}(x)+\varepsilon .
$$

Moreover, the application $\zeta:(0, t] \longrightarrow \mathbb{R}$ given by

$$
\zeta(s):=\frac{1}{s} \mathbb{E}\left[\int_{0}^{s} g\left(X_{r}^{x, u}\right) d r\right]
$$

for $s \in(0, t]$ is continuous. The definition of $T$ yields $\zeta(T) \geq V_{t}(x)+\varepsilon$. Finally, for every $0<s \leq t-T$,

$$
\frac{1}{s} \mathbb{E}\left[\int_{0}^{s} g\left(X_{r}^{x, u}\right) d r\right] \leq \frac{1}{s}\left((s+T)\left(V_{t}(x)+\varepsilon\right)-T \zeta(T)\right) \leq V_{t}(x)+\varepsilon .
$$

The proof of our proposition is now complete.

\section{The Uniform VANiShing APPROACH TO LONG RUN AVERAGING}

We are now able to state and prove the main result of our paper.

Theorem 4.1. Let us assume that $\left(v^{\delta}\right)_{\delta>0}$ is a relatively compact subset of $C\left(\mathbb{R}^{N} ;[0,1]\right)$. Then, for every $v \in C\left(\mathbb{R}^{N} ;[0,1]\right)$ and every sequence $\left(\delta_{m}\right)_{m \geq 1}$ such that $\lim _{m \rightarrow \infty} \delta_{m}=0$ and $\left(v^{\delta_{m}}\right)_{m \geq 1}$ converges uniformly to $v$ on $\mathbb{R}^{N}$, the following equality holds true

$$
\liminf _{t \rightarrow \infty} \sup _{x \in \mathbb{R}^{N}}\left|V_{t}(x)-v(x)\right|=0 .
$$

Remark 4.2. In particular, whenever $v^{\delta}$ converges to some $v^{*}$ uniformly on $\mathbb{R}^{N}$ as $\delta$ goes to 0 , the functions $V_{t}$ converge to $v^{*}$ uniformly on $\mathbb{R}^{N}$ as $t \rightarrow \infty$. Conversely, whenever $\left(v^{\delta}\right)_{\delta>0}$ is a relatively compact subset of $C\left(\mathbb{R}^{N} ;[0,1]\right)$, if $V_{t}$ converge to some $v^{*}$ uniformly on $\mathbb{R}^{N}$ as $t \rightarrow \infty$, then $v^{*}$ is the only limit point $\left(v^{\delta}\right)_{\delta>0}$ with respect to the usual topology on $C\left(\mathbb{R}^{N} ;[0,1]\right)$.

Proof. Let us fix $v \in C\left(\mathbb{R}^{N} ;[0,1]\right)$ and some sequence $\left(v^{\delta_{m}}\right)_{m \geq 1}$ converging uniformly to $v$ on $\mathbb{R}^{N}$.

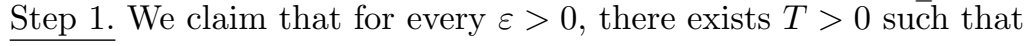

$$
V_{t}(x) \geq v(x)-\varepsilon,
$$

for all $t \geq T$ and all $x \in \mathbb{R}^{N}$.

We begin by fixing some $\varepsilon>0$. Our uniform convergence assumption yields the existence of some $m_{0} \geq 1$ such that

$$
\sup _{y \in \mathbb{R}^{N}}\left|v^{\delta_{m}}(y)-v(y)\right| \leq \frac{\varepsilon}{8}
$$

for all $m \geq m_{0}$. Let us fix $m \geq m_{0}$. Since $\lim _{T \rightarrow \infty} \int_{\frac{T \varepsilon}{4}}^{\infty} \delta_{m}^{2} s e^{-\delta_{m} s} d s=0$, there exists some $T>0$ for which

$$
\int_{\frac{S \varepsilon}{6}}^{\infty} \delta_{m}^{2} s e^{-\delta_{m} s} d s<\frac{\varepsilon}{8}
$$

for all $S \geq T$. 
Step 1.1. We reason by contradiction. Let us suppose that, for some $\varepsilon>0$, for every $T^{\prime}>0$ there exists some $t \geq T^{\prime}$ and some $x \in \mathbb{R}^{N}$ such that

$$
V_{t}(x)<v(x)-\varepsilon .
$$

In particular, one can find some $t \geq T$ satisfying (7). By proposition 3.2, one gets the existence of some admissible control process $u$ and some time horizon $0 \leq T_{0} \leq t\left(1-\frac{\varepsilon}{6}\right)$ such that

$$
\frac{1}{s} \mathbb{E}\left[\int_{T_{0}}^{s+T_{0}} g\left(X_{r}^{x, u}\right) d r\right] \leq V_{t}(x)+\frac{\varepsilon}{2}<v(x)-\frac{\varepsilon}{2},
$$

for every $0<s \leq \frac{t \varepsilon}{6}$. One notices that ( $\omega$-wise), the function $s \mapsto \phi(s):=\int_{0}^{s} g\left(X_{T_{0}+r}^{x, u}\right) d r$ is absolutely continuous on the compact set $\left[0, \frac{t \varepsilon}{6}\right]$. Also, its $(d s$-almost everywhere) derivative coincides $(d s$-a.e. $)$ with the càdlàg function $s \mapsto g\left(X_{s}^{x, u}\right)$. This equality should be understood $\mathbb{P}$ - a.e. In particular, using the integration by parts formula for absolutely continuous functions and taking expectation, we get

$$
\begin{aligned}
\delta_{m} \mathbb{E}\left[\int_{0}^{\frac{t \varepsilon}{6}} e^{-\delta_{m} s} g\left(X_{s+T_{0}}^{x, u}\right) d s\right] & =\delta_{m} e^{-\delta_{m} \frac{t \varepsilon}{6}} \mathbb{E}\left[\int_{T_{0}}^{T_{0}+\frac{t \varepsilon}{6}} g\left(X_{r}^{x, u}\right) d r\right] \\
& +\delta_{m}^{2} \mathbb{E}\left[\int_{0}^{\frac{t \varepsilon}{6}} e^{-\delta_{m} s} \int_{T_{0}}^{T_{0}+s} g\left(X_{r}^{x, u}\right) d r d s\right]
\end{aligned}
$$

Hence, using the inequalities (8) and (6), then recalling that $g \leq 1$, we have

$$
\begin{aligned}
\delta_{m} \mathbb{E}\left[\int_{0}^{\infty} e^{-\delta_{m} s} g\left(X_{s+T_{0}}^{x, u}\right) d s\right] & =\delta_{m} \mathbb{E}\left[\int_{0}^{\frac{t \varepsilon}{6}} e^{-\delta_{m} s} g\left(X_{s+T_{0}}^{x, u}\right) d s\right]+\delta_{m} \mathbb{E}\left[\int_{\frac{t \varepsilon}{6}}^{\infty} e^{-\delta_{m} s} g\left(X_{s+T_{0}}^{x, u}\right) d s\right] \\
& \leq \delta_{m}^{2} \mathbb{E}\left[\int_{0}^{\frac{t \varepsilon}{6}} s e^{-\delta_{m} s} \frac{1}{s} \int_{T_{0}}^{T_{0}+s} g\left(X_{r}^{x, u}\right) d r d s\right]+\frac{\varepsilon}{8} \\
& \leq v(x)-\frac{3 \varepsilon}{8} .
\end{aligned}
$$

Step 1.2. The monotonicity Proposition 3.1 and the choice of $\delta_{m}$ yield

$$
v(x)-\frac{\varepsilon}{8} \leq \mathbb{E}\left[v\left(X_{T_{0}}^{x, u}\right)\right]-\frac{\varepsilon}{8} \leq \mathbb{E}\left[v^{\delta_{m}}\left(X_{T_{0}}^{x, u}\right)\right] \leq \delta_{m} \mathbb{E}\left[\int_{0}^{\infty} e^{-\delta_{m} s} g\left(X_{s+T_{0}}^{x, u}\right) d s\right] .
$$

We recall that the inequality (9) holds true to finally get

$$
v(x)-\frac{\varepsilon}{8} \leq v(x)-\frac{3 \varepsilon}{8},
$$

which is an obvious contradiction. The first step is now complete.

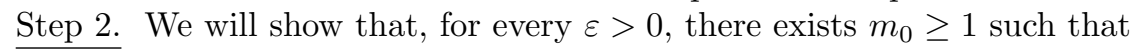

$$
V_{\delta_{m}^{-1}}(x) \leq v(x)+\varepsilon
$$

for all $m \geq m_{0}$ and all $x \in \mathbb{R}^{N}$.

Once again, we argue by contradiction. We assume that, for some $\varepsilon \in\left(0, \frac{2}{9}\right)$ and every $m_{1} \geq 1$, there exists some $m \geq m_{1}$ and some $x \in \mathbb{R}^{N}$ for which

$$
V_{\delta_{m}^{-1}}(x)>v(x)+\varepsilon .
$$


We define

$$
\eta(\varepsilon):=e^{-\left(1-\frac{\varepsilon}{2}\right)}\left(2-\frac{\varepsilon}{2}\right)-2 e^{-1}
$$

One notices that $\lim _{\varepsilon \rightarrow 0} \eta(\varepsilon)=0$. The uniform convergence assumption yields the existence of some $n_{0}$ such that

$$
v^{\delta_{m}}(y) \leq v(y)+\frac{\varepsilon \eta(\varepsilon)}{8}
$$

for all $m \geq n_{0}$ and all $y \in \mathbb{R}^{N}$. On the other hand, the inequality (5) yields the existence of some $T_{0}>n_{0}$ such that, for every $s \geq T_{0}$ and every $y \in \mathbb{R}^{N}, u \in \mathcal{U}$,

$$
\mathbb{E}\left[\frac{1}{s} \int_{0}^{s} g\left(X_{r}^{y, u}\right) d r\right] \geq V_{s}(y) \geq v(y)-\frac{\varepsilon \eta(\varepsilon)}{8} .
$$

Under our assumptions, for every $m_{1}>T_{0}$, one finds some $m \geq m_{1}$ and some $x_{m} \in \mathbb{R}^{N}$ such that, for every control $u \in \mathbb{L}^{0}\left(\mathbb{R}^{N} \times \mathbb{R}_{+} ; U\right)$,

$$
v\left(x_{m}\right)+\varepsilon<V_{\delta_{m}^{-1}}\left(x_{m}\right) \leq \delta_{m} \mathbb{E}\left[\int_{0}^{\delta_{m}^{-1}} g\left(X_{r}^{x_{m}, u}\right) d r\right] .
$$

In particular, for every admissible control process $u$ and every $\delta_{m}^{-1}\left(1-\frac{\varepsilon}{2}\right) \leq r \leq \delta_{m}^{-1}$,

$$
\begin{aligned}
\frac{1}{r} \mathbb{E}\left[\int_{0}^{r} g\left(X_{l}^{x_{m}, u}\right) d l\right] & =\frac{\delta_{m}^{-1}}{r}\left(\delta_{m} \mathbb{E}\left[\int_{0}^{\delta_{m}^{-1}} g\left(X_{l}^{x_{m}, u}\right) d l\right]\right)- \\
& -\frac{1}{r} \mathbb{E}\left[\int_{r}^{\delta_{m}^{-1}} g\left(X_{l}^{x_{m}, u}\right) d l\right] \\
& \geq \frac{\delta_{m}^{-1}}{r}\left(v\left(x_{m}\right)+\varepsilon\right)-\frac{\delta_{m}^{-1}-r}{r} \geq v\left(x_{m}\right)+\frac{7 \varepsilon}{16} .
\end{aligned}
$$

We recall that $g \geq 0$. For every admissible control process $u \in \mathbb{L}^{0}\left(\mathbb{R}^{N} \times \mathbb{R}_{+} ; U\right)$, using (14) and (13) and the integration by parts formula for absolutely continuous functions, one has, for every $R$ large enough,

$$
\begin{aligned}
\delta_{m} \mathbb{E}\left[\int_{0}^{R} e^{-\delta_{m} s} g\left(X_{s}^{x_{m}, u}\right) d s\right] & \geq \mathbb{E}\left[\int_{0}^{R} \delta_{m}^{2} s e^{-\delta_{m} s} \frac{1}{s} \int_{0}^{s} g\left(X_{r}^{x_{m}, u}\right) d r d s\right] \\
& \geq \int_{\delta_{m}^{-1}\left(1-\frac{\varepsilon}{2}\right)}^{\delta_{m}^{-1}} \delta_{m}^{2} s e^{-\delta_{m} s} \mathbb{E}\left[\frac{1}{s} \int_{0}^{s} g\left(X_{r}^{x_{m}, u}\right) d r\right] d s \\
& +\int_{0}^{R} 1_{\left(T_{0}, \infty\right) \backslash\left[\delta_{m}^{-1}\left(1-\frac{\varepsilon}{2}\right), \delta_{m}^{-1}\right]}(s) \delta_{m}^{2} s e^{-\delta_{m} s} \mathbb{E}\left[\frac{1}{s} \int_{0}^{s} g\left(X_{r}^{x_{m}, u}\right) d r\right] d s \\
& \geq\left(v\left(x_{m}\right)+\frac{7 \varepsilon}{16}\right) \omega\left(\delta_{m}, \delta_{m}^{-1}\right) \\
& +\left(v\left(x_{m}\right)-\frac{\varepsilon \eta(\varepsilon)}{8}\right) \int_{0}^{R} 1_{\left(T_{0}, \infty\right) \backslash\left[\delta_{m}^{-1}\left(1-\frac{\varepsilon}{2}\right), \delta_{m}^{-1}\right]}(s) \delta_{m}^{2} s e^{-\delta_{m} s} d s
\end{aligned}
$$

where $\omega(\delta, t):=e^{-\delta\left(t\left(1-\frac{\varepsilon}{2}\right)\right)}\left(\delta t\left(1-\frac{\varepsilon}{2}\right)+1\right)-e^{-\delta t}(\delta t+1)$. Taking $R \rightarrow \infty$, it follows that

$$
\delta_{m} \mathbb{E}\left[\int_{0}^{\infty} e^{-\delta_{m} s} g\left(X_{s}^{x_{m}, u}\right) d s\right] \geq\left(v\left(x_{m}\right)+\frac{7 \varepsilon}{16}\right) \eta(\varepsilon)+\left(v\left(x_{m}\right)-\frac{\varepsilon \eta(\varepsilon)}{8}\right)\left(e^{-T_{0} \delta_{m}}\left(\delta_{m} T_{0}+1\right)-\eta(\varepsilon)\right)
$$


where $\eta(\varepsilon)$ is given by (11). Hence, for $m>m_{1}$ such that $e^{-T_{0} \delta_{m}}\left(T_{0} \delta_{m}+1\right) \geq 1-\frac{\varepsilon \eta(\varepsilon)}{16}$,

$$
\begin{aligned}
\delta_{m} \mathbb{E}\left[\int_{0}^{\infty} e^{-\delta_{m} s} g\left(X_{s}^{x_{m}, u}\right) d s\right] & \geq\left(v\left(x_{m}\right)+\frac{7 \varepsilon}{16}\right) \eta(\varepsilon) \\
& +\left(v\left(x_{m}\right)-\frac{\varepsilon \eta(\varepsilon)}{8}\right)\left(\left(e^{-T_{0} \delta_{m}}\left(T_{0} \delta_{m}+1\right)-\eta(\varepsilon)\right)\right) \\
& \geq v\left(x_{m}\right)+\frac{7 \varepsilon \eta(\varepsilon)}{16}-\frac{\varepsilon \eta(\varepsilon)}{8}-\frac{\varepsilon \eta(\varepsilon)}{16} \geq v\left(x_{m}\right)+\frac{\varepsilon \eta(\varepsilon)}{4} .
\end{aligned}
$$

Since this inequality holds true for arbitrary $u \in \mathbb{L}^{0}\left(\mathbb{R}^{N} \times \mathbb{R}_{+} ; U\right)$, one gets

$$
v^{\delta_{m}^{-1}}\left(x_{m}\right) \geq v\left(x_{m}\right)+\frac{\varepsilon \eta(\varepsilon)}{4},
$$

which comes in contradiction with (12). The proof of our theorem is now complete.

\section{REFERENCES}

[1] A Almudevar. A dynamic programming algorithm for the optimal control of piecewise deterministic Markov processes. SIAM J. Control Optim., 40(2):525-539, AUG 302001.

[2] M. Arisawa. Ergodic problem for the Hamilton-Jacobi-Bellman equation. II. Annales de l'Institut Henri Poincare (C) Non Linear Analysis, 15(1):1 - 24, 1998.

[3] G. Barles and E. R. Jakobsen. On the convergence rate of approximation schemes for Hamilton-Jacobi-Bellman equations. ESAIM, Math. Model. Numer. Anal., 36(1):M2AN, Math. Model. Numer. Anal., 2002.

[4] R. Buckdahn, D. Goreac, and M. Quincampoix. Existence of asymptotic values for nonexpansive stochastic control systems. preprint, 2013.

[5] O. Costa. Average impulse control of piecewise deterministic processes. IMA Journal of Mathematical Control and Information, 6(4):375-397, 1989.

[6] O. L. V. Costa and F. Dufour. The vanishing discount approach for the average continuous control of piecewise deterministic Markov processes. Journal of Applied Probability, 46(4):1157-1183, DEC 2009.

[7] O. L. V. Costa and F. Dufour. Average continuous control of piecewise deterministic Markovprocesses. SIAM J. Control Optim., 48(7):4262-4291, 2010.

[8] M. H. A. Davis. Piecewise-deterministic Markov-processes - A general-class of non-diffusion stochastic-models. Journal of the Royal Statistical Society Series B-Methodological, 46(3):353-388, 1984.

[9] M. H. A. Davis. Control of Piecewise-deterministic processes via discrete-time dynamic-programming. Lect. Notes Control Inf. Sci., 78:140-150, 1986.

[10] M. H. A. Davis. Markov models and optimization, volume 49 of Monographs on Statistics and Applied Probability. Chapman \& Hall, London, 1993.

[11] M.A.H. Dempster and J.J. Ye. Generalized Bellman-Hamilton-Jacobi optimality conditions for a control problem with a boundary condition. Appl. Math. Optim., 33(3):211-225, MAY-JUN 1996.

[12] W. Feller. An Introduction to Probability Theory and its Applications Vol. II. New York: John Wiley \& Sons, 2nd edition, 1971.

[13] L. Forwick, M. Schal, and M. Schmitz. Piecewise deterministic Markov control processes with feedback controls and unbounded costs. Acta Appl. Math., 82(3):239-267, JUL 2004.

[14] D. Gatarek. Optimality conditions for impulsive control of piecewise-deterministic processes. Math. Control Signal Syst., $5(2): 217-232,1992$.

[15] D. Goreac. Viability, Invariance and Reachability for Controlled Piecewise Deterministic Markov Processes Associated to Gene Networks. ESAIM-Control Optimisation and Calculus of Variations, 18(2):401-426, APR 2012.

[16] D. Goreac and O.-S. Serea. Linearization Techniques for Controlled Piecewise Deterministic Markov Processes; Application to Zubov's Method. Applied Mathematics and Optimization, 66:209-238, 2012. 10.1007/s00245-012-9169-x.

[17] G. H. Hardy and J. E. Littlewood. Tauberian theorems concerning power series and dirichlet's series whose coefficients are positive. Proceedings of the London Mathematical Society, s2-13(1):174-191, 1914.

[18] N. V. Krylov. On the rate of convergence of finite-difference approximations for Bellman's equations with variable coefficients. Probab. Theory Related Fields, 117(1):1-16, 2000.

[19] M. Oliu-Barton and G. Vigeral. A uniform Tauberian theorem in optimal control. In P.Cardaliaguet and R.Cressman, editors, Annals of the International Society of Dynamic Games vol 12: Advances in Dynamic Games. Birkhäuser Boston, 2013.14 pages. 
[20] H. M. Soner. Optimal control with state-space constraint. II. SIAM J. Control Optim., 24(6):1110-1122, 1986. 